\title{
Compósitos à base de cimento e gesso reforçados com partículas de madeira de Eucalyptus grandis
}

\author{
Laércio Mesquita Júnior*, Douglas Lamounier Faria, José Benedito Guimarães Júnior, Tony Matheus \\ Carvalho Eugênio, Saulo Rocha Ferreira, Giovanni Francisco Rabelo
}

Departamento de Ciências Florestais, Programa de Pós-Graduação em engenharia de Biomateriais, Universidade Federal de Lavras, Lavras, MG, Brasil.

\begin{abstract}
RESUMO O objetivo do trabalho foi produzir e avaliar física e mecanicamente compósitos de gesso-madeira e cimento-madeira utilizando partículas de Eucalyptus grandis, afim de se analisar a possível substituição do cimento pelo gesso como aglutinante, visando reduzir o tempo de cura. Foi utilizado o gesso em pó comercial e partículas de madeira de Eucalyptus grandis, obtidas por moagem em moinho de martelos. Os painéis foram produzidos em moldes metálicos, com dimensões de $25 \times 25 \times 1,5 \mathrm{~cm}$ (LxLxA), densidade nominal de 1,2 g. $\mathrm{cm}^{-3}$ e uma proporção de 1:2,75 (madeira:gesso) e água-gesso de 1:0,4. Painéis de referência foram produzidos utilizando o cimento Portland CP V-ARI como aglutinante. Foi utilizado cloreto de cálcio $\left(\mathrm{CaCl}^{2}\right)$ como catalisador para acelerar a hidratação. Os resultados indicaram que os painéis não apresentaram diferenças estatísticas significativas na densidade, em absorção de água e inchamento em espessura ambos após 2 e $24 \mathrm{~h}$ de imersão em água, módulo de elasticidade para flexão e compressão paralela. Os painéis de cimento-madeira obtiveram maiores valores em retenção de umidade, módulo de ruptura para ensaio de flexão estática e ligação interna. Esses resultados demonstram que a utilização do gesso como aglutinante pode ser viável, sendo necessários mais estudos sobre a perda de resistência mecânica do gesso em contato com a umidade.
\end{abstract}

Palavras-chave: Painel gesso/madeira; propriedades físicas e mecânicas; análise química.

\section{Composites based on cement and plaster reinforced with wood particles of Eucalyptus grandis}

\begin{abstract}
The objective of this study was to produce and evaluate physically and mechanically composites of plaster-wood and cement-wood using particles of Eucalyptus grandis, in order to analyze the possible replacement of the cement by the gypsum as a binder, in order to reduce the cure time. The panels were produced in metal molds with dimensions of $25 \mathrm{x} 25 \mathrm{x}$ $1,5 \mathrm{~cm}$ (LxLxA), nominal density of $1,2 \mathrm{~g} . \mathrm{cm}^{-3}$ and a ratio of 1:2,75 (wood: plaster) and water-plaster of 1:0,4. Reference panels were produced using the CP V-ARI Portland cement as a binder. Calcium chloride $\left(\mathrm{CaCl}^{2}\right)$ was used as the catalyst to accelerate hydration. The results indicated that the panels did not show significant statistical differences in density, water absorption and swelling in thickness both after 2 and $24 \mathrm{~h}$ of immersion in water, modulus of elasticity for flexion and parallel compression. The cement-wood panels obtained higher values in moisture retention, modulus of rupture for static bending test and internal bonding. These results demonstrate that the use of gypsum as a binder may be feasible and further studies on the loss of mechanical resistance of gypsum in contact with moisture are required.
\end{abstract}

Keywords: Panel gypsum/wood; physical and mechanical properties; chemical analysis.

\section{Introdução}

Os painéis de cimento-madeira são fabricados a partir da mistura de partículas de madeira, um aglutinante mineral (cimento ou gesso), água e aditivos químicos (MENDES et al., 2011), com aplicação voltada a construção civil, principalmente na Europa e no Japão, em função de suas características adequadas para uso estrutural (IWAKIRI; PRATA, 2008).

Tais painéis são produzidos atualmente, quase que em sua totalidade, com as madeiras de Pinus (BERTOLINI et al., 
2014; CASTRO; IWAKIRI, 2014; GARCEZ et al., 2016; VILLAS-BÔAS et al., 2017) e de Eucalipto (CASTRO et al., 2014; GUIMARÃES JÚNIOR et al., 2016; IWAKIRI et al., 2013; MENDES et al., 2011; MESQUITA et al., 2015; RIOS et al., 2015).

Apesar de sua larga aceitação e utilização em mercados exigentes, como a Europa e a Ásia, esse tipo de painel ainda não está inserido comercialmente no mercado brasileiro (MENDES et al., 2011). Segundo CASTRO et al. (2014), O interesse por esse material deve-se principalmente às suas propriedades como isolantes térmicos, virtualmente incombustíveis, à resistência ao ataque de agentes biodegradadores, à alta estabilidade dimensional e à fácil trabalhabilidade.

Segundo Marra (1992), a relação com base no peso entre a madeira e o cimento mais empregada, inclusive industrialmente, é de 1:2,75. A madeira e o cimento são os principais componentes que formam a estrutura dos painéis cimento-madeira. Outras relações, maiores ou menores têm sido testadas, porém à medida que se aumenta a proporção de madeira em relação ao cimento, o tempo de cura dos painéis torna-se mais prolongado (LATORRACA; IWAKIRI, 2005).

Um dos problemas encontrados que afeta diretamente a produção de painéis de cimento-madeira é a composição química da madeira, a qual varia principalmente em função da espécie utilizada, influenciando no processo de cura e endurecimento do cimento. Segundo Simatupang et al. (1978), os extrativos presentes na madeira são os principais responsáveis pela inibição da solidificação do cimento, e segundo Beraldo et al. (2002), a presença de açúcares e extrativos solúveis em água ou álcali, retarda o tempo de pega do cimento.

Por outro lado, o gesso é um material de construção importante para aplicações em interiores, pois é barato e de fácil utilização. As placas de gesso apresentam boas propriedades, principalmente em termos de isolamento térmico e atendendo os padrões de segurança contra incêndio (SCHUG et al., 2017). Uma outra vantagem do gesso, é que seu endurecimento ou cura devido a hidratação do sulfato de cálcio hemi-hidratado $\left(\mathrm{CaSO}^{4} .0,5 \mathrm{H}^{2} \mathrm{O}\right)$ ocorre em 3 dias (JOHN; CINCOTTO, 2007). Contudo compósitos à base de gesso apresentam grande perda de resistência mecânica em contato com umidade, sendo indicado seu uso apenas em ambientes internos (PACHON-RODRIGUEZ et al., 2011).

Diante do exposto, o objetivo deste trabalho foi avaliar as propriedades físicas e mecânicas dos painéis de cimentomadeira e gesso-madeira utilizando partículas de Eucalyptus grandis e analisar a possível substituição do cimento pelo gesso como aglutinante, afim de reduzir o tempo de cura.

\section{Material e Métodos}

Foram utilizadas nesta pesquisa 4 clones de Eucalyptus grandis com vinte e oito anos de idade, oriundos de um plantio na Universidade Federal de Lavras - UFLA (Lavras, Minas Gerais). Para a formação dos painéis, utilizou-se como aglutinantes minerais o gesso em pó, o cimento Portland CP V-ARI de alta resistência inicial e o cloreto de cálcio como acelerador de cura do cimento.

Selecionadas e abatidas três árvores por clone de Eucalyptus grandis, em seguida, retirados discos nas porções: base, $25 \%, 50 \%, 75 \%$ e topo, de acordo com a altura do fuste de cada árvore. Para posterior determinação da densidade básica da madeira, utilizou-se da norma NBR 11941 (Associação Brasileira de Normas Técnicas - ABNT, 2003). Foi também determinada a razão de compactação dos painéis, obtida pela densidade dos painéis sobre a densidade da madeira. Determinou-se o teor de extrativos totais de acordo com a norma NBR 14853 (ABNT, 2010); o teor de lignina insolúvel, de acordo com a norma NBR 7989 (ABNT, 2010); o teor de cinzas, de acordo com a norma NBR 13999 (ABNT, 
2017), e o teor de holocelulose, obtido de acordo com o procedimento descrito por Browning (1963).

Após a coleta do Eucalyptus grandis, as toras foram acondicionadas imersas em um tanque à temperatura de 80 ${ }^{\circ} \mathrm{C}$ por 24 horas para amolecimento da madeira, em seguida laminadas em um torno laminador para geração das lâminas, secas à temperatura de $105{ }^{\circ} \mathrm{C}$ até massa constante. Posteriormente as lâminas de eucalipto foram trituradas em um moinho martelo, para a geração das partículas do tipo sliver, sendo estas submetidas a peneiramento mecânico. As partículas usadas na produção dos painéis foram aquelas que passaram pela peneira de $1,83 \mathrm{~mm}$ e ficaram retidas na de 1,69 $\mathrm{mm}$.

O delineamento experimental constituiu-se de dois tratamentos. Foi adotado delineamento inteiramente casualizado, sendo produzidos três painéis para o tratamento cimento-madeira e três painéis de gesso-madeira, totalizando
6 painéis de aglutinante-madeira de dimensões $48 \times 48 \times 1,5$ cm (comprimento, largura e espessura, respectivamente).

Após a pesagem de cada componente, estes foram misturados em uma betoneira para obtenção de massa homogênea. Retirada da betoneira, a massa de cada painel foi devidamente separada e distribuída aleatoriamente na caixa formadora do colchão, com as dimensões de $48 \times 48 \mathrm{~cm}$, untada com óleo diesel para facilitar a retirada do painel após sua prensagem e grampeamento. Após o carregamento dos painéis, a prensa foi fechada com pressão específica de $4 \mathrm{MPa}$ e temperatura ambiente, realizando-se em seguida o grampeamento dos painéis, ao qual permaneceram por um período de 24 horas. Após esse período, os grampos foram retirados e os painéis foram acondicionados em câmara climatizada à temperatura de $20 \pm 2{ }^{\circ} \mathrm{C}$ e umidade relativa de $65 \pm 3 \%$ durante 28 dias, sendo então realizados os ensaios das propriedades físicas e mecânicas dos painéis.

Tabela 1. Características da produção dos painéis.

Table 1. Production characteristics of the panels.

$\begin{array}{cc} & \text { TOTAL } \\ \text { Volume do Painel }\left(\mathrm{cm}^{3}\right) & 937,5 \\ \text { Relação madeira:aglutinante }(\mathrm{kg}: \mathrm{kg}) & 1: 2,75 \\ \text { Relação água:aglutinante } & 1: 0,4 \\ \text { Aditivo químico }(\%) & 4 \\ \text { Massa específica nominal do painel }\left(\mathrm{cm}^{3}\right) & 1,2 \mathrm{~g}\end{array}$

Tabela 2. Dimensões dos corpos de prova e normas utilizadas para os testes físico-mecânicos.

Table 2. Dimensions of the specimens and standards used for physical mechanical testing.

\begin{tabular}{ccc}
\hline Propriedade avaliada & Norma utilizada & Dimensões dos corpos de prova \\
\hline Densidade & & $5 \times 5 \mathrm{~cm}$ \\
\hline $\begin{array}{c}\text { Absorção de água 2 e 24 horas } \\
\text { Inchamento em espessura 2 e 24 horas }\end{array}$ & ASTM $1037(2016)$ & $15 \times 15 \mathrm{~cm}$ \\
\hline Ligação interna & & $5 \times 5 \mathrm{~cm}$ \\
\hline Compressão paralela & ASTM $1037(2016)$ & $25 \times 10 \mathrm{~cm}$ \\
\hline Módulo de elasticidade à flexão & ASTM $1037(2016)$ & $25 \times 5 \mathrm{~cm}$
\end{tabular}


O processo de obtenção dos corpos de prova foi realizado com auxílio de uma serra circular. Foram retirados de cada painel, dois corpos de prova para absorção de água e inchamento em espessura, seis corpos de prova para ligação interna, quatro corpos de prova para compressão paralela e quatro corpos de prova para flexão estática. As dimensões dos corpos de prova e as normas utilizadas estão representadas na Tabela 2.

Foram realizados os seguintes testes físicos: densidade do painel, absorção de água (AA) após 2 e 24 horas e inchamento em espessura (IE) após 2 e 24 horas. Os testes mecânicos realizados foram: ligação interna, módulo de elasticidade à flexão estática (MOE) e módulo de ruptura à flexão estática (MOR).

Para avaliar as propriedades físicas e mecânicas dos painéis aglutinante-madeira produzidos, os dados foram submetidos à análise de variância e o teste de média de ScottKnott, a 5\% de significância. Os dados foram avaliados estatisticamente com o uso do software Sisvar.

Foram utilizados também, como base para comparação dos resultados, os valores mínimos das propriedades mecânicas de painéis comerciais, estabelecidos pelo processo Bison (BISON WOOD-CEMENT BOARD, 1978).

\section{Resultados e Discussão}

$\mathrm{Na}$ Tabela 3, estão apresentados os valores médios da densidade básica, teor de extrativos totais, lignina insolúvel, holocelulose e cinzas da madeira.

A densidade básica obtida para o Eucalyptus grandis foi de $0,61 \mathrm{~g} . \mathrm{cm}^{-3}$. Este valor influencia na quantidade de partícula empregada na formação do painel, pois está diretamente relacionado ao seu peso. Madeiras com densidades mais elevadas resultam em um baixo grau de compactação e como consequência geram um painel de baixa qualidade (SILVA et al., 2005). Sorfa; Bongers (1982), destacam que além da composição do painel, a densidade apresenta alta influência na resistência de compósitos cimento-madeira.

Os valores de densidade são idênticos aos obtidos por Iwakiri et al. (2008), que, estudando diferentes espécies de eucalipto, obtiveram valores médios de 0,60 g. $\mathrm{cm}^{-3}$ para Eucalyptus grandis.

O valor de extrativos totais alcançados foi de 6,12\%. Mori et al. (2007) encontraram para a madeira de Eucalyptus grandis um valor médio de $4 \%$ de extrativos totais. Verificase que o resultado obtido é similar ao encontrado ao da literatura.

Os extrativos totais são de extrema importância, pois, de acordo com Iwakiri et al. (2005), a capacidade de solidificação do cimento na presença da madeira é determinada pela composição química da mesma, sendo que os extrativos são os principais responsáveis pela inibição da solidificação do cimento. Isso implica influências diretas nas propriedades físicas e mecânicas dos painéis cimento-madeira. A presença de maiores quantidades de minerais e alguns extrativos apolares podem resultar no bloqueio de grupos químicos reativos para a adesão com adesivos polares, afetando, assim, a qualidade da colagem e o desempenho mecânico das chapas reconstituídas (NDAZI et al., 2007).

$\mathrm{Na}$ Tabela 4, estão apresentados os valores médios da densidade do painel, assim como a razão de compactação dos painéis produzidos.

O valor médio de densidade básica a $12 \%$ de umidade da madeira de Eucalyptus grandis utilizada nessa pesquisa foi $0,61 \mathrm{~g} \cdot \mathrm{cm}^{-3}$. Com relação às densidades médias obtidas para os painéis, estas foram um pouco inferiores em comparação às densidades nominais de $1,20 \mathrm{~g} . \mathrm{cm}^{-3}$ estabelecida no delineamento experimental. Tais diferenças podem ser atribuídas sobretudo às condições operacionais relacionadas à perda de materiais durante a formação do colchão e prensagem dos painéis, além do retorno em espessura do pai- 
Tabela 3. Caracterização da madeira de Eucalyptus grandis.

Table 3. Characterization of Eucalyptus grandis wood.

\begin{tabular}{cccccc}
\hline Tratamento & $\begin{array}{c}\text { Densidade básica } \\
\left({\left.\mathrm{g} . \mathrm{cm}^{-3}\right)}^{\text {Eucalyptus }}\right.\end{array}$ & Extrativos (\%) & Lignina (\%) & Holocelulose (\%) & Cinzas (\%) \\
\hline grandis & $0,61^{(0,30)}$ & $6,12^{(0,54)}$ & $28,02^{(1,24)}$ & $65,68^{(0,90)}$ & $0,18^{(0,02)}$ \\
\hline
\end{tabular}

Valores entre parênteses representam o desvio padrão

Tabela 4. Valores médios da densidade do painel, e razão de compactação dos painéis produzidos.

Table 4. Mean values of panel density, and compaction ratio of panels produced.

\begin{tabular}{ccc}
\hline Painel & Densidade do painel $\left(\mathrm{g} \cdot \mathrm{cm}^{-3}\right)$ & Razão de compactação \\
\hline Gesso/Madeira & $1,11^{(0,04)} \mathrm{A}$ & $1,81^{(0,03)} \mathrm{A}$ \\
Cimento/Madeira & $1,10^{(0,07)} \mathrm{A}$ & $1,80^{(0,04)} \mathrm{A}$ \\
\hline
\end{tabular}

Médias seguidas da mesma letra não diferem entre si pelo teste Scott-knott a 5\% de significância. Valores entre parênteses representam o desvio padrão.

nel após a sua retirada da prensa. Esses fatores influenciam diretamente na redução da densidade do painel em função da redução no peso de partículas do colchão e aumento da espessura e volume do painel.

Com relação a razão de compactação, pode-se observar que esta não apresentou variação. Segundo Scatolino et al. (2017), a baixa densidade das partículas é um dos fatores fundamentais para utilização de materiais lignocelulósicos na produção de painéis reconstituídos, com isso uma maior razão de compactação pode resultar em melhores propriedades mecânicas.

$\mathrm{Na}$ Tabela 5, estão apresentados os valores médios de absorção de água e inchamento em espessura, ambos após 2 e 24 horas de imersão em água.

Com relação à absorção de água e inchamento em espessura após 2 e 24 horas de imersão em água, não houve diferenças estatísticas entre os tratamentos. Iwakiri; Prata (2008), ao avaliarem as propriedades de painéis minerais de Eucalyptus grandis e Eucalyptus dunnii encontraram valores inferiores aos obtidos neste trabalho, com valor de absorção de água em 24 horas de 15,15\%, e inchamento em espessura em 24 horas de 2,26\%. Os valores médios de absorção de água após 24 horas obtidos nesse estudo para painéis cimentomadeira estão dentro da faixa de 15,69 a 22,22\%, encontrados por Latorraca (2000) para quatro espécies de eucalipto; já para inchamento em espessura, os valores médios obtidos para os painéis gesso-madeira estão um pouco acima da faixa de 1,64 a 2,12\%. Cabe ressaltar que os painéis de Eucalyptus grandis produzidos apresentaram valores médios próximos aos encontrados na literatura e do valor referencial máximo de $1,8 \%$ do processo Bison (BISON WOOD-CEMENT BOARD, 1978).

$\mathrm{Na}$ Tabela 6 estão apresentados os valores médios de MOE e MOR para o ensaio de flexão simples, compressão paralela e ligação interna.

Os resultados de MOE apresentados acima, demonstram que os painéis produzidos com madeira de Eucalyptus grandis apresentaram valores médios estatisticamente iguais em comparação aos tratamentos realizados. Cabe destacar ainda que, em termos de médias absolutas, os painéis de Eucalyptus grandis produzidos com cimento apresentaram a maior média entre os tratamentos. Na comparação com os valores mencionados na literatura, os valores médios de $\mathrm{MOE}$ obtidos para os tratamentos com madeira de Eucalyptus grandis foram superiores em relação ao valor mínimo referencial do processo Bison (BISON WOOD-CEMENT BOARD, 1978), que é de 2,94 MPa. Os resultados foram também satisfatórios em comparação aos valores encontra- 
Tabela 5. Absorção de água e inchamento em espessura após 2 e 24 horas de imersão em água.

Table 5. Water absorption and thickness swelling after 2 and 24 hours immersion.

\begin{tabular}{ccccc}
\hline Painel & $\begin{array}{c}\text { Absorção de água 2h } \\
(\%)\end{array}$ & $\begin{array}{c}\text { Absorção de água } \\
24 \mathrm{~h}(\%)\end{array}$ & $\begin{array}{c}\text { Inchamento em } \\
\text { espessura 2h (\%) }\end{array}$ & $\begin{array}{c}\text { Inchamento em } \\
\text { espessura 24h }(\%)\end{array}$ \\
\hline Gesso-madeira & $21,88^{(2,67)} \mathrm{A}$ & $26,67^{(4,10)} \mathrm{A}$ & $1,83^{(1,53)} \mathrm{A}$ & $2,67^{(0,79)} \mathrm{A}$ \\
Cimento-madeira & $20,43^{(0,86)} \mathrm{A}$ & $21,88^{(1,00)} \mathrm{A}$ & $1,37^{(0,68)} \mathrm{A}$ & $2,10^{(0,79)} \mathrm{A}$ \\
\hline
\end{tabular}

Médias seguidas da mesma letra não diferem entre si pelo teste Scott-knott a $5 \%$ de significância. Valores entre parênteses representam o desvio padrão.

Tabela 6. Módulos de elasticidade (MOE), ruptura (MOR), compressão e ligação interna.

Table 6. Modulus of elasticity (MOE), rupture (MOR), compressive strength and internal bond.

\begin{tabular}{ccccc}
\hline Painel & MOE $(\mathrm{MPa})$ & MOR $(\mathrm{MPa})$ & Compressão (MPa) & Ligação Interna (MPa) \\
\hline Gesso-madeira & $4329,78^{(1618,94)} \mathrm{A}$ & $6,08^{(3,23)} \mathrm{A}$ & $5,44^{(1,11)} \mathrm{A}$ & $0,53^{(0,09)} \mathrm{A}$ \\
Cimento-madeira & $6706,06^{(2516,76)} \mathrm{A}$ & $14,39^{(4,12)} \mathrm{B}$ & $4,70^{(1,08)} \mathrm{A}$ & $0,80^{(0,11)} \mathrm{B}$ \\
\hline
\end{tabular}

Médias seguidas da mesma letra não diferem entre si pelo teste Scott-knott a $5 \%$ de significância. Valores entre parênteses representam o desvio padrão.

dos por Iwakiri; Prata (2008), para painéis produzidos com madeiras de Eucalyptus grandis e Eucalyptus dunnii, cujas médias variaram na faixa de 0,98 a 4,78 $\mathrm{MPa}$.

Os valores médios de MOR, apresentados na Tabela 6, indicam que há diferença estatisticamente significativa entre os tratamentos. Guimarães et al. (2015), estudando a utilização da madeira de Eucalyptus grandis x Eucalyptus camaldulensis na produção de painéis cimento-madeira, obtiveram valores para MOR entre 15 e $19 \mathrm{MPa}$ e, portanto, superiores aos encontrados neste trabalho.

O valor mínimo estabelecido, para MOR pelo processo Bison (BISON WOOD-CEMENT BOARD, 1978) é de 8,92 $\mathrm{MPa}$, dessa forma, os painéis gesso-madeira avaliados apresentaram valores inferiores ao mínimo prescrito por este processo. Os resultados também foram inferiores em comparação aos valores obtidos por Iwakiri; Prata (2008), para painéis produzidos com madeiras de Eucalyptus grandis e Eucalyptus dunnii, cujas médias variaram na faixa de 3,53 a 9,90 MPa.

Os resultados apresentados indicam que a resistência à compressão paralela dos painéis gesso-madeira confeccionados foi estatisticamente igual aos painéis cimento-madeira. Santos et al. (2008) trabalhando com resíduos da madeira de Eremanthus erythropappus para produção de painéis cimento-madeira obtiveram valores médios de 7,28 $\mathrm{MPa}$ de resistência à compressão paralela dos painéis, ou seja, os resultados obtidos neste trabalho encontram-se próximos aos encontrados na literatura.

De acordo com os resultados observados para ligação interna, pode-se constatar que os painéis produzidos com cimento-madeira apresentaram valor médio de ligação interna estatisticamente maior em relação aos painéis gessomadeira. O valor médio de 0,53 e 0,80 MPa para os painéis de gesso-madeira e cimento-madeira, respectivamente, foram superiores ao valor mínimo requerido pelo processo Bison (BISON WOOD-CEMENT BOARD, 1978) que é de 0,39 MPa.

\section{Conclusões}

Com base nos resultados obtidos, as seguintes conclusões podem ser apresentadas:

Com relação às propriedades físicas, os painéis de gessomadeira mostram valores estatisticamente iguais aos de cimento-madeira;

$\mathrm{Na}$ flexão estática, os materiais estudados se mostram iguais (homogêneos) apenas no módulo de elasticidade 
(MOE), sendo que no módulo de ruptura (MOR) o painel cimento-madeira apresentou valor superior ao painel gessomadeira, uma vez que o painel gesso-madeira não se enquadrou as exigências mínimas do processo de Bison Wood-Cement board (1978);

Para ligação interna, todos os painéis se adequam aos requisitos mínimos descritos no processo de Bison WoodCement board (1978), porém os painéis de cimento-madeira apresentaram maior valor médio;

Para compressão paralela os resultados foram estatisticamente iguais e próximos aos valores encontrados na literatura.

Diante dos resultados obtidos, é viável a utilização do gesso como aglutinante em painéis minerais, porém se faz necessário o seu emprego em ambiente interno, sem contato com a água.

\section{Referências}

American Society for Testing Materials. ASTM D-1037-12: Standard Test Methods for Evaluating Properties of WoodBase Fiber and Particle Panel Materials, ASTM International, West Conshohocken, PA; 2016.

Associação Brasileira de Normas Técnicas. NBR 11941: Madeira - Determinação da densidade básica. Rio de Janeiro; 2003.

Associação Brasileira de Normas Técnicas. NBR 13999: Papel, cartão, pastas celulósicas e madeira: determinação do resíduo (cinza) após a incineração a $525^{\circ} \mathrm{C}$. Rio de Janeiro; 2017.

Associação Brasileira de Normas Técnicas. NBR 7989: Pasta celulósica e madeira: determinação de lignina insolúvel em ácido. Rio de Janeiro; 2010.

Associação Brasileira de Normas Técnicas. NBR 14853: Madeira: determinação do material solúvel em etanoltolueno e em diclorometano e em acetona. Rio de Janeiro; 2010.

BERALDO, A. L.; ARRUdA, A. C.; STANCATO, A. C.; SAMPAIO, C. A. P.; FERNANDES FILHO, O. P.; LEONEL, V. M. Compósitos à base de resíduos vegetais e cimento
Portland. In: Encontro Brasileiro em Madeiras e em Estruturas de Madeira, 2002.

BERTOLINI, M. S.; NASCIMENTO, M. F.; CHRISTOFORO, A. L.; LAHR, F. A. R. Painéis de partículas provenientes de rejeitos de Pinus sp. tratado com preservante CCA e resina derivada de biomassa. Revista Árvore, v. 38, n. 2, p. 339-346, 2014.

BISON WOOD-CEMENT BOARD. Bison-report. [S.1.],1978. $10 \mathrm{p}$.

CASTRO, V. G.; BRAZ, R. L.; AZAMBUJA, R. F.; LOIOLA, P. L.; IWAKIRI, S.; MATOS, J. L. M. Painéis cimento-madeira de Eucalyptus saligna com diferentes aditivos químicos e métodos de formação. Floresta, v. 45, n. 2, p. 349-360, 2015.

CASTRO, V.; ARAÚJO, R. D.; PARCHEN, C.; IWAKIRI, S. Evaluation of pretreatment effects of Eucalyptus benthamii Maiden \& Cambage wood on the compatibility degree with Portland cement. Revista Árvore, v. 38, n. 5, p. 935-942, 2014.

CASTRO, V.; IWAKIRI, S. Influência de diferentes níveis de acetilação nas propriedades físico-mecânicas de aglomerados e painéis madeira-cimento. Cerne, v. 20, n. 4, p. 535-540, 2014.

GARCEZ, M. R.; SANTOS, T.; GARCEZ, E. O.; GATTO, D. A. Propriedades físicas de compósitos cimento-madeira com serragem de Pinus elliottii tratada. Revista Ciência da Madeira, v. 7, n. 2, p. 70-80, 2016.

GUIMARÃES JÚNIOR, J. B.; MENDES, R. F.; GUIMARÃES, I. L.; LISBOA, F. N.; PROTÁSIO, T. P.; MENDES, L. M. Qualidade de painéis OSB (Oriented Strand Board) produzidos com a madeira de clones de Eucalyptus urophylla. Revista Ciência da Madeira, v. 7, n. 3, p. 163-169, 2016.

GUIMARÃES, J. C. O.; LIMA, J. T. C.; NUNES, J. S.; LISBOA, F. J. N.; GUIMARÃES JÚNIOR, J. B. Eficiência do tratamento de partículas de madeira de eucalipto do híbrido de Eucalyptus grandis X Eucalyptus camaldulensis para produção de painéis minerais. Enciclopédia biosfera, Centro Científico Conhecer - Goiânia, v.11, n.21; p.949, 2015.

IWAKIRI, S.; TRIANOSKI, R.; CUNHA, A. B.; PRATA, J. G.; HARA, M.; BILA, N. F. et al. Propriedades tecnológicas de painéis cimento-madeira produzidos com partículas de eucalipto. Revista de Ciências Agroveterinárias, v. 14, n. 3, p. 217-223, 2013. 
IWAKIRI, S.; ALBUQUERQUE, C. E. C.; PRATA, J. G.; COSTA, A. C. B. Utilização de madeiras de Eucalyptus grandis e Eucalyptus dunnii para produção de painéis de partículas orientadas - OSB. Ciência Florestal, Santa Maria, v. 18, n. 2, p. 265-270, abr.-jun., 2008.

IWAKIRI, S.; KEINERT JUNIOR, S.; ALBUQUERQUE, C. E. C. de; LATORRACA, J. V. de F.; MENDES, L. M. Painéis de madeira reconstituída. Curitiba: Ajir, 2005. v. 1, 247 p.

JOHN, V. M.; CINCOTTO, M. A. Gesso de construção civil. ISAIA, G. C. Materiais de construção civil e princípios de ciência e engenharia de materiais. IBRACON 727-760, 2007.

LATORRACA, J. V. F. Eucalyptus spp. na produção de painéis de cimento-madeira. 2000. 191 p. Tese (Doutorado em Ciências Florestais) - Universidade Federal do Paraná, Curitiba, 2000.

LATORRACA, J. V. F.; IWAKIRI, S. Painéis de cimentomadeira. In: IWAKIRI, S. (Ed.). Painéis de madeira reconstituída. Curitiba: FUPEF, 2005. p. 229-254.

MARRA, A. A. Technology of wood bonding: principles and pratice. New York: V. N. Reinhold, 1992. 453 p.

MESQUITA, R. G. A.; MENDES, L. M.; MENDES, R. F.; TONOLI, G. H. D.; MARCONCINI, J. M. Inclusão de feixes de sisal na produção de painéis MDP de eucalipto. Scientia Forestalis, v. 43, n. 10, p. 75-82, 2015.

MENDES, L. M.; LOSCHI, F. A. P.; PAULA, L. E. R.; MENDES, R. F.; GUIMARÃES JÚNIOR, J. B.; MORI, F. A. Potencial de utilização da madeira de clones de Eucalyptus urophylla na produção de painéis cimento-madeira. Cerne, v. 17, n. 1, p. 69-75, 2011.

MORI, F. A.; LOPES, Y. L. V.; MENDES, L. M.; LATORRACA, J. V. F. Estudo da compatibilidade entre a madeira e as cascas de Eucalyptus grandis e cimento Portland. Ciência Florestal, Santa Maria, v. 17, n. 3, p. 257-264, 2007.

NDAZI, B. S.; KARLSSON, S.; TESHA, J. V.; NYAHUMWA, C. W. Chemical and physical modifications of rice husks for use as composite panels. Composites Part A: Applied Science and Manufacturing, v.38, n. 33, p.925-935, 2007.

NORMEN FÜR HOLZFASERPLATEN SPANPLATTEN SPERRHOLZ: testing of wood chipboards, bending test, determination of bending strength. DIN 52362. 1982. p. 3940.
PACHON-RODRIGUEZ E.A.; GUILLON E.; HOUVENAGHEL G.; COLOMBANI J. ; Pressure solution as origin of the humid creep of a mineral material, Phys. Rev. E Stat. Nonlinear Soft Matter Phys. 84 (2011).

RIOS, P. D.; VIEIRA, H. C.; STUPP, A. M.; KNIESS, D. D.C.; BORBA, M. H.; CUNHA, A. B. Avaliação física e mecânica de painéis reconstituídos compostos por partículas de galhos secos de Araucaria angustifolia (Bertol.) Kuntze e madeira de Eucalyptus grandis Hill ex Maiden. Scientia Forestalis, v. 43, n. 106, p. 283-289, 2015.

SANTOS, R. C.; MENDES, L. M.; MORI, F. A.; MENDES, R. F. Aproveitamento de resíduos da madeira de candeia (Eremanthus erythropappus) para produção de painéis cimento-madeira. Cerne, Lavras, v. 14, n. 3, p. 241-250, 2008.

SCATOLINO, M. V.; COSTA, A. O.; GUIMARÃES JUNIOR, J. B.; PROTÁSIO, T. P.; MENDES, R. F.; MENDES, L. M. Eucalyptus wood and coffee parchment for particleboard production: physical and mechanical properties. Ciência e Agrotecnologia, v. 41, n. 2, p. 139-146, 2017.

SCHUG, B.; MANDEL, K.; SCHOTTNER, G.; SHMELIOV, A.; NICOLOSI, V.;BAESE, R.; PIETSCHMANN, B.; BIEBL, M.; SEXTL, G.; A mechanism to explain the creep behavior of gypsum plaster. Cement and Concrete Research v. 98, p. 122-12, 2017.

SILVA, G. C.; LATORRACA, J. V. F.; TEIXEIRA, D. E.; BORTOLETTO JÚNIOR, G. Produção do compósito madeira e casca de Eucalyptus urophylla S.T. Blake e cimento Portland. Scientia Forestalis, n. 68, p. 59-67, 2005.

SIMATUPANG, M. H.; SCHWARZ, G. H.; BROKER, F. W. Small scale plants for the manufacture of mineral-bonded wood composites. In: WORLD FORESTRY CONGRESS, 21p, 1978.

SORFA, P.; BONGERS, J. Wood-cement composite building units: part 1. Pretoria: National Timber Research Institute, 24 p, 1982.

VILLAS-BÔAS, B. T.; PARCHEN, C. F. A.; IWAKIRI, S; PRATA, J. G.; COSTA, M. R. M. M. Different effects assessment of dosages water/limer in the compressive strength of wood-cement composites. Revista Matéria, v. 22, n. 1, 2017. 